\title{
Laudatio für Jutta Limbach aus Anlass der feierlichen Verleihung der Ehrendoktorwürde des Fachbereichs Rechtswissenschaft der Universität Bremen
}

Meine sehr geehrten Damen und Herren, liebe Kollegen und Kolleginnen, vor allem aber: sehr verehrte, liebe Frau Limbach!

Sie sind die erste Frau, der die Ehrendoktorwürde des Fachbereichs Rechtswissenschaft der Universität Bremen verliehen wird, und dieses „erste“ findet sich in vielen Kapiteln Ihres Lebens wieder.

Als Juristen und Juristinnen sind wir es ja gewohnt, Allgemeine und Besondere Teile voneinander $\mathrm{zu}$ unterscheiden, und so gibt es heute Abend eine allgemeine Laudatio, mit der ich gerade begonnen habe, und eine besondere Laudatio, die Herr Rinken halten wird.

In meinem Teil der Laudatio will ich ein Juristinnenleben nachzeichnen, das exemplarisch zu nennen naheliegt - doch exemplarisch im Sinne von „,beispielhaft“ gleich typisch für viele kann es gar nicht sein (eben deswegen, weil Jutta Limbach an so vielen Stellen ihres Lebens die erste oder doch eine der ersten Juristinnen war). Exemplarisch im Sinne von „,beispielgebend“ hingegen sehr wohl. Vor allem für die nachfolgenden Juristinnengenerationen, aber nicht nur diese.

Frau Limbach hatte das Glück - wie sie für ein 1982 erschienenes Buch von Juristinnen über Juristinnen zu Protokoll gegeben hat ${ }^{1}$-, in einem Elternhaus aufzuwachsen, in dem „Bildung und Berufstätigkeit sowie soziales und politisches Engagement ... als selbstverständliche Rechte und Möglichkeiten von Frauen betrachtet [wurden], die es nur zu nutzen galt". Politisches Engagement hatten ihr bereits eigene Vorfahrinnen vorgelebt. Eine Urgroßmutter, Pauline Staegemann, war im 19. Jahrhundert, als Frauen jede politische Betätigung verboten war, ${ }^{2}$ trotzdem politisch aktiv, hatte in Berlin mehrmals Vereine für Arbeiterinnen gegründet und ist dafür ebenso oft zu Haftstrafen verurteilt worden, die sie auch abgesessen hat. ${ }^{3}$ Deren Tochter Elfriede, verheiratete Ryneck, die Großmutter väterlicherseits, wird in historischen Quellen bereits als Politikerin bezeichnet - zu Recht: 1919/20 war Elfriede Ryneck Mitglied der Weimarer Nationalversammlung, von 1920 bis 1924 Mitglied des Reichstages, von 1925 bis 1933 Mitglied

1 Fabricius-Brand, Margarete / Berghahn, Sabine / Sudhölter, Kristine (Hrsg.): Juristinnen: Berichte, Fakten, Interviews, Berlin-West: Elefanten-Press-Verlag, 1982, S. 178-181 (179).

2 Gerhard, Ute: Grenzziehungen und Überschreitungen: Die Rechte der Frauen auf dem Weg in die politische Öffentlichkeit, in: Dies. (Hrsg.): Frauen in der Geschichte des Rechts, München: Beck, 1997, S. 509-546 (524 ff.).

3 Juchacz, Marie: Sie lebten für eine bessere Welt: Lebensbilder führender Frauen des 19. und 20. Jahrhunderts, Berlin etc.: Dietz, 1971, S. 25-28; Wachenheim, Hedwig: Vom Großbürgertum zur Sozialdemokratie: Memoiren einer Reformistin, Berlin: Colloquium Verlag, 1973, S. 151 f. Seit 2004 verleiht die Arbeitsgemeinschaft sozialdemokratischer Frauen in der SPD Brandenburg zweijährlich einen nach Pauline Staegemann benannten Preis „für Zivilcourage, Einsatz für Gleichberechtigung und gesellschaftliche Gerechtigkeit“. 
des Preußischen Landtages, ${ }^{4}$ außerdem zeitweise Mitglied des Parteivorstandes der SPD.

Die dunkle Zeit des sogenannten Dritten Reichs haben Sie, Frau Limbach, als 1934 Geborene als Kind erlebt, in einem dezidiert sozialdemokratischen Elternhaus sicher nicht ohne Ängste. Prägender waren dann aber die Jugendjahre. Rechtswissenschaft als Studienfach wählte Jutta Limbach jedoch nicht, um die Arbeit ihrer Urgroßmutter und ihrer Großmutter fortzusetzen, sondern aus dem Wunsch heraus, eine solide Grundlage für eine spätere Tätigkeit als politische Journalistin zu erwerben. Sie studierte in Berlin, ihrem Geburtsort, und in Freiburg/Breisgau, bestand 1958 das Erste juristische Staatsexamen und 1962 die Große juristische Staatsprüfung (Anmerkung für die Jüngeren im Publikum: damals dauerte das Referendariat noch dreieinhalb Jahre) - und gleich danach wurde sie wissenschaftliche Assistentin am Fachbereich Rechtswissenschaft der Freien Universität Berlin. Ihr Journalismus-Traum war schon während des Jurastudiums verflogen, wie Frau Limbach einmal gesagt hat, weil ihr die Jurisprudenz ,einfach Spaß gemacht hat". 5 (Und in die Zunft der Schreibenden ist sie ja trotzdem eingetreten.) 1966 promovierte sie mit der Dissertation „,Theorie und Wirklichkeit der $\mathrm{GmbH}^{\prime}$, die als Band 2 der von Ernst E. Hirsch ${ }^{6}$ begründeten Schriftenreihe mit dem (heutigen) Titel „Schriftenreihe zur Rechtssoziologie und Rechtstatsachenforschung“ veröffentlicht wurde. Die rechtssoziologische Sichtweise behielt Frau Limbach auch in ihrer Habilitationsschrift bei. „Das gesellschaftliche Handeln, Denken und Wissen im Richterspruch“ heißt die Arbeit, mit der sie sich Ende 1971 für die Fächer Bürgerliches Recht, Handels- und Wirtschaftsrecht sowie Rechtssoziologie habilitierte. Gleich im Anschluss, Anfang 1972, trat sie die Professur für Bürgerliches Recht, Handels- und Wirtschaftsrecht und Rechtssoziologie an der Freien Universität Berlin an.

Dies sieht wie eine völlig normal verlaufene wissenschaftliche Karriere aus, und war doch alles andere. Lassen Sie mich an dieser Stelle einen kleinen professionshistorischen Exkurs machen. Zum Studium wurden Frauen, wie mittlerweile weithin bekannt ist, in

4 Bundesarchiv, „Akten der Reichskanzlei. Weimarer Republik” online, Biographien: Ryneck, Elfriede = www.bundesarchiv.de/aktenreichskanzlei/1919-1933/0011/adr/adrmr/kap1_6/para2_209.html (14.12.2008). Vgl. ferner Datenbank der Reichstagsabgeordneten, Basis: Reichstagshandbücher $(1919-1933 / 38)=$ mdz1.bib-bvb.de/ rt/select.html?name=Ryneck++Elfriede\&recherche $=$ ja (14.12.2008).

5 Jutta Limbach, DIE ZEIT, Nr. 37 vom 8.9.2005, S. 68.

6 Am 28.11.2008, einen Tag nach der Verleihung der Ehrendoktorwürde an Jutta Limbach, wurde eine Neuausgabe der zuerst 1982 unter dem Titel ,Aus des Kaisers Zeiten durch die Weimarer Republik in das Land Atatürks" im Schweitzer-Verlag erschienenen Autobiographie Hirschs, nunmehr unter dem Titel „Als Rechtsgelehrter im Lande Attatürks“, Berliner WissenschaftsVerlag, in einer Veranstaltung der Freien Universität präsentiert; Jutta Limbach als eine der prominentesten Schülerinnen Hirschs hielt einen der Vorträge. 
Deutschland erst zu Beginn des 20. Jahrhunderts zugelassen. ${ }^{7}$ Das Jurastudium konnten die ersten Frauen aber nur mit der Promotion abschließen; denn zu den Staatsexamina wurden sie erst Anfang der 1920er Jahre zugelassen. Auch war mit der Öffnung der Universitäten für Studentinnen zunächst keineswegs eine Öffnung akademischer Laufbahnen verbunden; denn zu habilitieren war ihnen verwehrt, bis der Preußische Minister für Wissenschaft, Kunst und Volksbildung - auf eine Eingabe von Edith Stein hin - in einem Erlass vom 21.2.1920 Steins Auffassung beitrat, „,dass in der Zugehörigkeit zum weiblichen Geschlecht kein Hindernis gegen die Habilitation erblickt werden darf". 8 Die erste und bis Kriegsende einzige Habilitation einer Juristin in Deutschland ${ }^{9}$ gab es $^{\circ}$ Ende 1932 in Hamburg. ${ }^{10}$ Im späteren Westdeutschland wurde eine Juristin 1946 habilitiert, ${ }^{11}$ zwischen 1959 und 1970 folgten sechs weitere, ${ }^{12}$ d.h. Frau Limbach war erst die achte in Deutschland (West) habilitierte Juristin. ${ }^{13}$ (Von 1946 bis 1977 wurden in-

7 Gemeinhin wird 1908 als das entscheidende Jahr angegeben. Dieses Jahr markiert aber nur die Zulassung von Frauen zum Universitätsstudium in Preußen. In den anderen deutschen Ländern öffneten sich die Universitätstore für Frauen zwischen 1900 und 1918; vgl. Rinken, Alfred: Einführung in das juristische Studium: Juristenausbildung und Juristenpraxis im Verfassungsstaat, 3. Aufl., München: Beck, 1996, S. 47 f.; Soden, Kristine von: Auf dem Weg in die Tempel der Wissenschaft: Zur Durchsetzung des Frauenstudiums im Wilhelminischen Deutschland, in: Gerhard, Ute (Hrsg.): Frauen in der Geschichte des Rechts, München: Beck, 1997, S. 617-632. Einige der ganz frühen deutschen Juristinnen hatten in Zürich studiert, dessen Universität bereits seit Ende der 1860er Jahre Frauen als Studentinnen zugelassen hatte; vgl. Verein Feministische Wissenschaft Schweiz / Katharina Belser / Gabi Einsele / Rachel Gratzfeld / Regula Schnurrenberger (Hrsg.): Ebenso neu als kühn: 120 Jahre Frauenstudium an der Universität Zürich, Zürich: eFeF, 1988.

8 Zitiert nach Boedeker, Elisabeth / Meyer-Plath, Maria: 50 Jahre Habilitation von Frauen in Deutschland, Göttingen: Verlag Otto Schwartz \& Co., 1974, S. 5.

9 Die Schweizerin Emily Kempin-Spyri (1853-1901) war von der Universität Zürich bereits 1891 ,ausnahmsweise“ habilitiert worden; vgl. Hasler, Eveline: Die Wachsflügelfrau, München: dtv, 1994. S. 197 f.

10 Magdalene Schoch; vgl. Boedeker/Meyer-Plath (Fn. 8), S. 191. Die dort genannten bibliographischen Angaben enthalten jedoch einige Fehler. Inzwischen ist die Biographie dieser bemerkenswerten Frau gut aufgearbeitet; vgl. Nicolaysen, Rainer: Für Recht und Gerechtigkeit: Über das couragierte Leben der Juristin Magdalene Schoch (1897-1987), Zeitschrift des Vereins für Hamburgische Geschichte 2006 (92. Jg.), S. 113-143. Zu den am Beispiel Schoch aufgezeigten Schwierigkeiten, durch die NS-Zeit verschüttetes Wissen über frühe Juristinnen wiederzufinden, vgl. Plett, Konstanze: The loss of early women lawyers from collective memory in Germany - and the difficulty to rediscover them: A memoir of Magdalena Schoch, in: Karstedt, Susanne (Hrsg.): Legal Institutions and Collective Memories, Oxford: Hart Publishing, 2009.

11 Gerda Krüger, die 1938 zum Rücktritt vom abgeschlossenen Habilitationsverfahren aus politischen Gründen genötigt worden war; Boedeker/Meyer-Plath (Fn. 8), S. 191 f. Möglicherweise handelte es sich jedoch um eine philosophische Habilitation, da in Boedeker/MeyerPlath beim Namen der Zusatz „Dr. jur. Dr. phil. habil.“ angegeben ist.

12 Dies waren nach Boedeker/Meyer-Plath (Fn. 8), S. 192 ff.: Anne-Eva Brauneck 1959, Marie Luise Hilger 1959, Hilde Kaufmann 1961, Ilse Staff 1969, Marianne Bauer 1969, Brigitte Keuk 1970.

13 Da Boedeker/Meyer-Plath einerseits nur Dozentinnen befragt haben, andererseits nur den Zeitraum bis 1970 erfassen, ist nicht ganz auszuschließen, dass ihnen die eine oder andere Habilitation einer weiteren Juristin entgangen ist; aber dies ist doch sehr unwahrscheinlich. 
teressanterweise in der - viel kleineren - DDR neun Juristinnen habilitiert. ${ }^{14}$ Noch überschaubarer sind die Berufungen von Frauen an juristische Fakultäten: In der alten Bundesrepublik gab es die erste $1965,{ }^{15}$ die zweite $1966,{ }^{16}$ die dritte $1971^{17}$ und die vierte im Januar 1972 - diese ist die von Frau Limbach. (Nur zur Vervollständigung des Bildes: In der DDR gab es 1971 immerhin schon neun juristische Hochschullehrerinnen. ${ }^{18}$ )

Zurück zu den Lebens-Stationen von Jutta Limbach. Wer einmal auf rechtssoziologische Fragestellungen gestoßen ist, den und die lässt diese Sichtweise nicht mehr los. So ist es auch nicht verwunderlich, dass Frau Limbach zu der Gruppe von Wissenschaftlern gehörte, die 1976 die „Vereinigung für Rechtssoziologie“ 19 gründeten, deren Vorstand sie mehrere Jahre angehörte und deren Mitglied sie bis heute ist. Im rechtssoziologischen Zusammenhang steht auch ihre im Auftrag des Bundesjustizministeriums erstellte empirische Studie „Die gemeinsame Sorge geschiedener Eltern in der Rechtspraxis“, veröffentlicht $1989 ;{ }^{20}$ Zweck dieser Studie war die Vorbereitung einer Gesetzesänderung zum elterlichen Sorgerecht, die nach einer Entscheidung des Bundesverfassungsgerichts im Jahre $1982^{21}$ erforderlich geworden war. ${ }^{22}$

Im Wintersemester 1981/82 weilte Frau Limbach hier in Bremen zur Wahrnehmung einer Gastprofessur, die vornehmlich der Mitwirkung an einem Forschungsprojekt des Rechtssoziologen Rüdiger Lautmann diente - ein Projekt, das in den 1980er Jahren zu einer in verschiedenen Fachzeitschriften ausgetragenen Kontroverse über das Rechtsbewusstsein von Frauen geführt hatte, ${ }^{23}$ an der sich Frau Limbach allerdings nicht beteiligte. Hingegen war sie hinfort und ist bis heute an der feministischen Rechtsdebatte beteiligt. Dies dokumentiert sich u.a. in dem 1988 gemeinsam mit Ute Gerhard herausgegebenen Band „Rechtsalltag von Frauen“ ${ }^{24}$ in dem sie selbst „Engagement und Distanz als Probleme einer feministischen Rechtswissenschaft" ${ }^{\prime 25}$ ausgemacht hat. Außerdem ist Frau Limbach von Anbeginn an Mitherausgeberin der wegen der Umschlagfarbe so genannten „Lila Reihe“, der „Schriften zur Gleichstellung der Frau“, in der von 1991

14 Boedeker/Meyer-Plath (Fn. 8), S. 333-336, 362. Aus der Namensliste, die auch nicht habilitierte Dozentinnen enthält, wurden nur die habilitierten gezählt.

15 Anne-Eva Brauneck (1910-2007), Gießen, Professur für Strafrecht und Kriminologie.

16 Hilde Kaufmann (1920-1981), Kiel, ab 1970 Köln.

17 Ilse Staff (1928 geboren), Frankfurt am Main.

18 Wie Fn. 13. Von diesen war nur Anita Grandke nach der deutschen Einigung noch tätig (an der Humboldt-Universität zu Berlin, bis 1994).

19 www.rechtssoziologie.info (28.12.2008).

20 Erschienen im Bundesanzeiger-Verlag, Köln, in der Reihe „Rechtstatsachenforschung“.

21 BVerfGE 61, 358.

22 Der Entscheidung des Bundesverfassungsgerichts zur elterlichen Sorge von 1982 wurde durch Gesetzesänderung erst mit dem Gesetz zur Reform des Kindschaftsrechts (Kindschaftsrechtsreformgesetz - KindRG) vom 16.12.1997 (BGBl. I S. 2942) Rechnung getragen, das am 1.7.1998 in Kraft trat.

23 Das Projekt selbst, das unter Leitung von Rüdiger Lautmann mit Jutta Limbach und Ute Gerhard durchgeführt wurde, galt der Erforschung der Folgen und Auswirkungen der Eherechtsreform von 1976. Die Diskussion um das Rechtsbewusstsein von Frauen, auf die hier Bezug genommen wird, hatte sich aus diesem Projekt heraus entwickelt, war aber davon unabhängig.

24 Bei Suhrkamp, Frankfurt am Main, erschienen (edition suhrkamp 1423).

25 S. $169-182$. 
bis jetzt 32 Bände erschienen sind. ${ }^{26}$ Zudem hat sie zur Gleichberechtigung von Frauen zahlreiche veröffentlichte und unveröffentlichte Vorträge gehalten; allen zur Lektüre empfehlen kann ich z.B. ihren vor einem Jahr in Karlsruhe gehaltenen Vortrag über „50 Jahre Gleichberechtigungsgesetz“ .27

Wer jetzt jedoch glaubt, Jutta Limbachs wissenschaftlicher Umgang sei mit der „Frauenszene“ erschöpft, den kann ich beruhigen. Stellvertretend für viele wissenschaftliche Aktivitäten sei ihre Beteiligung an der Wiederbelebung der „Kritischen Vierteljahresschrift für Gesetzgebung und Rechtswissenschaft", kurz: KritV, genannt, die von 1859 bis 1919 in drei Folgen erschienen war. 1986 beginnt die vierte Folge, und dieser Neubeginn wurde maßgeblich von dem Bremer Kollegen Eike Schmidt betrieben. Frau Limbach ist auch hier von Anbeginn bis heute Mitherausgeberin. (Um die Leseempfehlungen um zwei zu ergänzen: Der „Neuerscheinungbegründungsbeitrag“ von Eike Schmidt ${ }^{28}$ liest sich als hochmodernes Ausbildungsprogramm, und die Rezensionsabhandlung von Jutta Limbach ${ }^{29}$ im ersten [Doppel-]Heft gibt an drei Beispielen hervorragende Nachhilfe in juristischer Methodik - beides auch 22 Jahre nach Erscheinen noch höchst lesenswert.)

All diese Tätigkeiten belegen, dass Frau Limbach der Wissenschaft keinesfalls den Rücken gekehrt hat, als sie Rufen auf andere Positionen im Staat folgte. Von 1989 bis 1994 war sie Senatorin für Justiz des Landes Berlin. In diesem Amt war sie ebenfalls die erste Frau - allerdings keineswegs die einzige und nicht einmal die einzige Rechtsprofessorin im Senat; denn mit Heide Pfarr hatte der damalige Regierende Bürgermeister $^{30}$ sich einer weiteren Frau dieser Qualifikation versichert (und insgesamt sogar einen Senat mit mehr Senatorinnen als Senatoren gebildet). Als Frau Limbach ihr Amt im März 1989 antrat, war noch nicht absehbar, was in ihre Amtszeit fallen würde: die Vereinigung der beiden Deutschlands und der beiden Teile Berlins - gerade für die Justizsenatorin gewiss keine einfache, dafür aber ebenso gewiss eine höchst aufregende Zeit, auch wenn nicht alle ihre Vorschläge in der Gemeinsamen Verfassungskommission zur

26 Im Nomos-Verlag, Baden-Baden. Davon stammen fünf Bände von Bremer Wissenschaftlern und Wissenschaftlerinnen, darunter der von Ursula Rust herausgegebene 14. Band (Juristinnen an den Hochschulen - Frauenrecht in Lehre und Forschung, 1997), dessen Einleitungsbeitrag ,Juristinnen im Wissenschaftsbetrieb - Feminisierung der Jurisprudenz?“ Jutta Limbach verfasst hat.

27 Auf die Verfügbarkeit im Internet hat mich mein Mitlaudator aufmerksam gemacht: http:// www.gleichberechtigung-goes-online.de/doc/doc_download.cfm?uuid=70264466C2975CC8AC756DC31D7261AB\&\&IRACER_AUTOLINK\&\& (26.12.2008).

28 Schmidt, Eike: Gesetzgebung, Rechtspraxis und Jurisprudenz als Gegenstände kritischer Betrachtung (oder vom Berufe unserer Zeit, eine sich kritisch nennende Rechtszeitschrift wiederzubegründen), KritV 1986 (69. Jg.), S. 1-20.

29 Limbach, Jutta: Die Kompensation von Ungleichgewichtslagen: Eine Rezensionsabhandlung, KritV 1986 (69. Jg.), S. 165-188.

30 Walter Momper. Jutta Limbach blieb aber auch im zweiten Senat Diepgen ab 1991 Senatorin für Justiz. 
Reform des Grundgesetzes, die 1992/93 zusammengetreten war und deren ordentliches Mitglied sie war, die erforderlichen Mehrheiten fanden. ${ }^{31}$

Den Berliner Senat verließ Frau Limbach im März 1994, um das höchste in diesem Staat zu vergebende Amt anzutreten, für das die Qualifikation als „,Volljurist/in“ erforderlich ist: nämlich um Richterin des Bundesverfassungsgerichts in Karlsruhe zu werden, wobei sie sofort auch Vizepräsidentin wurde und klar war, dass sie ab Herbst desselben Jahres Präsidentin sein würde. Auch hier kann ich mir eine kleine professionshistorische Anmerkung nicht versagen. Für viele Jahre gab es jeweils nur eine Richterin am Bundesverfassungsgericht, was zu dem Scherzwort vom „Schneewittchen-Senat“ führte für denjenigen der beiden Senate, dem diese Richterin angehörte: eine Frau und sieben Männer. Als Frau Limbach, an das Bundesverfassungsgericht kam, gab es dort allerdings schlagartig sogar einen höheren Frauenanteil als in der Justiz im Übrigen: $30 \%$ gegenüber $25 \%$; denn zugleich waren weitere Frauen in dieses Amt gewählt worden. ${ }^{32}$

Die Würdigung der Karlsruher Zeit wird verabredungsgemäß Herr Rinken übernehmen, so dass ich in das Jahr 2002 springe, als Frau Limbach aus Altersgründen aus diesem Amt schied, aber alles andere tat, als sich zur Ruhe zu setzen; denn abermals nahtlos übernahm sie eine neue Aufgabe, diesmal als Präsidentin des Goethe-Instituts, ein Amt, das sie als ,das schönste Ehrenamt, das die Bundesrepublik Deutschland zu vergeben hat" bezeichnet hat. ${ }^{33}$ Bei Betrachtung ihrer Publikationsliste ist unverkennbar, dass Frau Limbach in diesem Amt einen neuen Gegenstand für publizistische Betätigung entdeckt hat: die Sprache. Die von ihr herausgegebenen Bücher über aus- und eingewanderte Wörter ${ }^{34}$ und das schönste deutsche Wort ${ }^{35}$ sind eine Fundgrube für alle Sprachinteressierten. In ihrer in diesem Jahr erschienenen Monographie „Hat Deutsch eine Zukunft? Unsere Sprache in der globalisierten Welt" ${ }^{* 36}$ zieht Jutta Limbach ein Stück weit die Summe ihres beruflichen Lebens; aus diesem Buch spricht für mich sowohl die Juristin als auch die Politikerin als auch die Kulturbotschafterin.

31 Vgl. hierzu die Laudatio von Alfred Rinken (in diesem Heft); ferner Limbach, Jutta / EckertzHöfer, Marion (Hrsg.): Frauenrechte im Grundgesetz des geeinten Deutschland: Diskussion in der Gemeinsamen Verfassungskommission von Bundestag und Bundesrat und der Bundesratskommission Verfassungsreform - Dokumentation -, Baden-Baden: Nomos, 1993 (Schriften zur Gleichstellung der Frau, 7).

32 Aktuell ist der Anteil allerdings wieder gesunken: 3 von 16 macht nur noch knapp $20 \%$, und der Erste Senat ist tatsächlich wieder zum „Schneewittchen-Senat“ geworden.

33 In der Widmung des Buches Limbach, Jutta: Hat Deutsch eine Zukunft? Unsere Sprache in der globalisierten Welt, München: Beck, 2008; die vollständige Widmung lautet: „Dieses Buch ist als ein bescheidener Dank für das schönste Ehrenamt gedacht, das die Bundesrepublik Deutschland zu vergeben hat."

34 Limbach, Jutta (Hrsg.): Ausgewanderte Wörter: Eine Auswahl der interessantesten Beiträge zur internationalen Ausschreibung „Ausgewanderte Wörter“, Ismaning: Hueber, 2007; Limbach, Jutta (Hrsg.): Eingewanderte Wörter: Eine Auswahl der schönsten Beiträge zum internationalen Wettbewerb „Wörter mit Migrationshintergrund - das beste eingewanderte Wort“, Ismaning: Hueber, 2008.

35 Limbach, Jutta (Hrsg.): Das schönste deutsche Wort: Liebeserklärungen an die deutsche Sprache, Freiburg im Breisgau etc.: Herder, 2006.

36 Vgl. Fn. 33. 
Schließen möchte ich mit einer persönlichen und einer dienstlichen Anmerkung.

Die persönliche: Bei unseren sporadischen Begegnungen in den letzten zwanzig Jahren (deren erste übrigens auch hier in Bremen war, als ich eine Vortragsveranstaltung der Juristischen Gesellschaft mit Ihnen, Frau Limbach, zum Thema „Feministische Jurisprudenz" moderieren durfte) habe ich mich immer wieder gefreut, dass Sie Ihre Verbundenheit mit der feministischen Sache nie in Abrede gestellt haben (und ich weiß uns einig darin, dass diese Sache ja überhaupt nicht an das Geschlecht ihrer Verfechterinnen und - ja, auch: - Verfechter ${ }^{37}$ geknüpft ist).

Die dienstliche: Von vielen Seiten hat das amtierende Dekanat Lob dafür bekommen, dass wir Sie zur Verleihung der Ehrendoktorwürde vorgeschlagen haben, obgleich einige dachten, dass dies eine Ehrung sei, bei der die Universität Bremen eigentlich nur zu spät kommen könne. Umso größer war das Erstaunen (auch mein eigenes), dass Sie zwar schon einige Ehrendoktorwürden erhalten haben, aber bislang ausschließlich von ausländischen Universitäten. ${ }^{38}$ Deshalb freuen wir uns ganz besonders, Frau Limbach, dass Sie zur Annahme dieser Auszeichnung bereit sind und so der Universität Bremen Gelegenheit geben, ihr Eintreten für „Gleichberechtigung der Geschlechter“ als eines der universitären Leitziele ${ }^{39}$ unter Beweis zu stellen.

37 Hier sei nur an einige namhafte Feministen mit ihren Werken erinnert: Theodor Gottlieb von Hippel, Über die bürgerliche Verbesserung der Weiber, 1793 (Nachdruck 1977); John Stuart Mill, The subjection of women, 1869 (dt. 1869 als „Auszug aus John Stuart Mill's Unterordnung der Frauen“, aber auch schon vollständig unter dem seither verwendeten Titel „Die Hörigkeit der Frau“, letzter Nachdruck 1997); August Bebel, Die Frau und der Sozialismus, 1883 (letzter Nachdruck 1994); Bernard Shaw, The intelligent woman's guide to socialism and capitalism, 1928 (dt. Übers.: Wegweiser für die intelligente Frau zum Sozialismus und Kapitalismus, 1928, Neuausgabe 1978).

381999 Basel, 2002 Rotterdam und University College London, 2003 York University Toronto und 2003 Peru.

39 „Gleichberechtigung der Geschlechter“ gehört seit 2000 dazu, vgl. die Dokumentation im Bremer Uni Schlüssel (BUS) Nr. 60, Oktober/Novermber 2000, S. 10 f.; die Leitziele sind auch unter http://www.uni-bremen.de/campus/leitziele.php3 (26.12.2008) zu finden. 